\title{
Growth estimates for modified Neumann integrals in a half-space
}

\author{
Yudong Ren ${ }^{1 *}$ and Pai Yang ${ }^{2}$
}

${ }^{\text {*Correspondence: }}$ yudong83@163.com

1 Department of Mathematics and Information Science, Henan

University of Economics and Law Zhengzhou, 450002, P.R. China Full list of author information is available at the end of the article

\begin{abstract}
Our aim in this paper is to deal with the growth properties for modified Neumann integrals in a half-space of $\mathbf{R}^{n}$. As an application, the solutions of Neumann problems in it for a slowly growing continuous function are also given.
\end{abstract}

Keywords: Dirichlet problem; harmonic function; half-space

\section{Introduction and main results}

Let $\mathbf{R}$ and $\mathbf{R}_{+}$be the sets of all real numbers and of all positive real numbers, respectively. Let $\mathbf{R}^{n}(n \geq 3)$ denote the $n$-dimensional Euclidean space with points $x=\left(x^{\prime}, x_{n}\right)$, where $x^{\prime}=\left(x_{1}, x_{2}, \ldots, x_{n-1}\right) \in \mathbf{R}^{n-1}$ and $x_{n} \in \mathbf{R}$. The boundary and closure of an open set $\Omega$ of $\mathbf{R}^{n}$ are denoted by $\partial \Omega$ and $\bar{\Omega}$, respectively. For $x \in \mathbf{R}^{n}$ and $r>0$, let $B_{n}(x, r)$ denote the open ball with center at $x$ and radius $r$ in $\mathbf{R}^{n}$.

The upper half-space is the set $H=\left\{\left(x^{\prime}, x_{n}\right) \in \mathbf{R}^{n}: x_{n}>0\right\}$, whose boundary is $\partial H$. For a set $F, F \subset \mathbf{R}_{+} \cup\{0\}$, we denote $\{x \in H ;|x| \in F\}$ and $\{x \in \partial H ;|x| \in F\}$ by $H F$ and $\partial H F$, respectively. We identify $\mathbf{R}^{n}$ with $\mathbf{R}^{n-1} \times \mathbf{R}$ and $\mathbf{R}^{n-1}$ with $\mathbf{R}^{n-1} \times\{0\}$, writing typical points $x, y \in \mathbf{R}^{n}$ as $x=\left(x^{\prime}, x_{n}\right), y=\left(y^{\prime}, y_{n}\right)$, where $y^{\prime}=\left(y_{1}, y_{2}, \ldots, y_{n-1}\right) \in \mathbf{R}^{n-1}$. Let $\theta$ be the angle between $x$ and $\hat{e}_{n}$, i.e., $x_{n}=|x| \cos \theta$ and $0 \leq \theta<\pi / 2$, where $\hat{e}_{n}$ is the $i$ th unit coordinate vector and $\hat{e}_{n}$ is normal to $\partial H$.

We shall say that a set $E \subset H$ has a covering $\left\{r_{j}, R_{j}\right\}$ if there exists a sequence of balls $\left\{B_{j}\right\}$ with centers in $H$ such that $E \subset \bigcup_{j=0}^{\infty} B_{j}$, where $r_{j}$ is the radius of $B_{j}$ and $R_{j}$ is the distance between the origin and the center of $B_{j}$.

For positive functions $g_{1}$ and $g_{2}$, we say that $g_{1} \lesssim g_{2}$ if $g_{1} \leq M g_{2}$ for some positive constant $M$. Throughout this paper, let $M$ denote various constants independent of the variables in question. Further, we use the standard notations, $[d]$ is the integer part of $d$ and $d=[d]+\{d\}$, where $d$ is a positive real number.

Given a continuous function $f$ in $\partial H$, we say that $h$ is a solution of the Neumann problem in $H$ with $f$, if $h$ is a harmonic function in $H$ and

$$
\lim _{x \in H, x \rightarrow y^{\prime}} \frac{\partial}{\partial x_{n}} h(x)=f\left(y^{\prime}\right)
$$

for every point $y^{\prime} \in \partial H$.

For $x \in \mathbf{R}^{n}$ and $y^{\prime} \in \mathbf{R}^{n-1}$, consider the kernel function

$$
K_{n}\left(x, y^{\prime}\right)=-\frac{\beta_{n}}{\left|x-y^{\prime}\right|^{n-2}},
$$

○2013 Ren and Yang; licensee Springer. This is an Open Access article distributed under the terms of the Creative Commons Attribution License (http://creativecommons.org/licenses/by/2.0), which permits unrestricted use, distribution, and reproduction in any medium, provided the original work is properly cited. 
where $\beta_{n}=2 /(n-2) \sigma_{n}$ and $\sigma_{n}$ is the surface area of the $n$-dimensional unit sphere. It has the expression

$$
K_{n}\left(x, y^{\prime}\right)=\sum_{k=0}^{\infty} \frac{|x|^{k}}{|y|^{n+k-2}} C_{k}^{\frac{n-2}{2}}\left(\frac{x \cdot y^{\prime}}{|x|\left|y^{\prime}\right|}\right)
$$

where $C_{k}^{\frac{n}{2}}(t)$ is the ultraspherical (Gegenbauer) polynomials [1]. The series converges for $\left|y^{\prime}\right|>|x|$, and each term in it is a harmonic function of $x$.

The Neumann integral is defined by

$$
N[f](x)=\int_{\partial H} K_{n}\left(x, y^{\prime}\right) f\left(y^{\prime}\right) d y^{\prime},
$$

where $f$ is a continuous function on $\partial H, \alpha_{n}=2 / n \sigma_{n}$ and $\sigma_{n}=\pi^{\frac{n}{2}} / \Gamma\left(1+\frac{n}{2}\right)$ is the volume of the unit $n$-ball.

The Neumann integral $N[f](x)$ is a solution of the Neumann problem on $H$ with $f$ if (see $[2$, Theorem 1 and Remarks])

$$
\int_{\partial H} \frac{f\left(y^{\prime}\right)}{\left(1+\left|y^{\prime}\right|\right)^{n-2}} d y^{\prime}<\infty
$$

In this paper, we consider functions $f$ satisfying

$$
\int_{\partial H} \frac{\left|f\left(y^{\prime}\right)\right|^{p}}{\left(1+\left|y^{\prime}\right|\right)^{n+\alpha-2}} d y^{\prime}<\infty
$$

for $1 \leq p<\infty$ and $\alpha \in \mathbf{R}$.

For this $p$ and $\alpha$, we define the positive measure $\mu$ on $\mathbf{R}^{n}$ by

$$
d \mu\left(y^{\prime}\right)= \begin{cases}\left|f\left(y^{\prime}\right)\right|^{p}\left|y^{\prime}\right|^{-n-\alpha+2} d y^{\prime}, & y^{\prime} \in \partial H(1,+\infty), \\ 0, & Q \in \mathbf{R}^{n}-\partial H(1,+\infty) .\end{cases}
$$

If $f$ is a measurable function on $\partial H$ satisfying (1.1), we remark that the total mass of $\mu$ is finite.

Let $\epsilon>0$ and $\delta \geq 0$. For each $x \in \mathbf{R}^{n}$, the maximal function $M(x ; \mu, \delta)$ is defined by

$$
M(x ; \mu, \delta)=\sup _{0<\rho<\frac{|x|}{2}} \frac{\mu\left(B_{n}(x, r)\right)}{\rho^{\delta}} .
$$

The set $\left\{x \in \mathbf{R}^{n} ; M(x ; \mu, \delta)>\epsilon\right\}$ is denoted by $E(\epsilon ; \mu, \delta)$.

To obtain the Neumann solution for the boundary data $f$, as in [3-6], we use the following modified kernel function defined by

$$
L_{n, m}\left(x, y^{\prime}\right)= \begin{cases}-\beta_{n} \sum_{k=0}^{m-1} \frac{|x|^{k}}{|y|^{n+k-2}} C_{k}^{\frac{n-2}{2}}\left(\frac{x \cdot y^{\prime}}{|x| y^{\prime} \mid}\right), & \left|y^{\prime}\right| \geq 1 m \geq 1, \\ 0, & \left|y^{\prime}\right|<1 m \geq 1, \\ 0, & m=0\end{cases}
$$

for a non-negative integer $m$. 
For $x \in \mathbf{R}^{n}$ and $y^{\prime} \in \mathbf{R}^{n-1}$, the generalized Neumann kernel is defined by

$$
K_{n, m}\left(x, y^{\prime}\right)=K_{n}\left(x, y^{\prime}\right)-L_{n, m}\left(x, y^{\prime}\right) \quad(m \geq 0)
$$

Since $|x|^{k} C_{k}^{\frac{n-2}{2}}\left(\frac{x \cdot y^{\prime}}{|x|\left|y^{\prime}\right|}\right)(k \geq 0)$ is harmonic in $H$ (see [4]), $K_{n, m}\left(\cdot, y^{\prime}\right)$ is also harmonic in $H$ for any fixed $y^{\prime} \in \partial H$. Also, $K_{n, m}\left(x, y^{\prime}\right)$ will be of order $\left|y^{\prime}\right|^{-(n+m-2)}$ as $y^{\prime} \rightarrow \infty$ (see [7, Theorem D]).

Put

$$
N_{m}[f](x)=\int_{\partial H} K_{n, m}\left(x, y^{\prime}\right) f\left(y^{\prime}\right) d y^{\prime},
$$

where $f$ is a continuous function on $\partial H$. Here, note that $N_{0}[f](x)$ is nothing but the Neumann integral $N[f](x)$.

The following result is due to Siegel and Talvila (see [5, Corollary 2.1]). For similar results with respect to the Schrödinger operator in a half-space, we refer readers to papers by $\mathrm{Su}$ (see [8]).

Theorem A Iff is a continuous function on $\partial H$ satisfying (1.1) with $p=1$ and $\alpha=m$, then

$$
\lim _{|x| \rightarrow \infty, x \in H} N_{m}[f](x)=o\left(|x|^{m} \sec ^{n-2} \theta\right)
$$

The next result deals with a type of uniqueness of solutions for the Neumann problem on $H$ (see [9, Theorem 3]).

Theorem B Let l be a positive integer and $m$ be a non-negative integer. Iff is a continuous function on $\partial H$ satisfying

$$
\int_{\partial H} \frac{\left|f\left(y^{\prime}\right)\right|}{\left(1+\left|y^{\prime}\right|\right)^{n+m-2}} d y^{\prime}<\infty
$$

and $h$ is a solution of the Neumann problem on $H$ with $f$ such that

$$
\lim _{|x| \rightarrow \infty, x \in H} h^{+}(x)=o\left(|x|^{l+m}\right)
$$

then

$$
h(x)=N_{m}[f](x)+\Pi\left(x^{\prime}\right)+\sum_{j=1}^{\left[\frac{l+m}{2}\right]} \frac{(-1)^{j}}{(2 j) !} x_{n}^{2 j} \Delta^{j} \Pi\left(x^{\prime}\right)
$$

for any $x=\left(x^{\prime}, x_{n}\right) \in H$, where $h^{+}(x)$ is the positive part of $h$,

$$
\Delta^{j}=\left(\frac{\partial^{2}}{\partial x_{1}^{2}}+\frac{\partial^{2}}{\partial x_{2}^{2}}+\cdots+\frac{\partial^{2}}{\partial x_{n-1}^{2}}\right) \quad(j=1,2 \ldots)
$$

and $\Pi\left(x^{\prime}\right)$ is a polynomial of $x^{\prime} \in \mathbf{R}^{n-1}$ of degree less than $l+m$. 
Our first aim is to be concerned with the growth property of $N_{m}[f]$ at infinity and establish the following theorem.

Theorem 1 Let $1 \leq p<\infty, 0 \leq \beta \leq(n-2) p, n+\alpha-2>-(n-1)(p-1)$ and

$$
\begin{aligned}
& 1-\frac{1-\alpha}{p}<m<2-\frac{1-\alpha}{p} \quad \text { if } p>1, \\
& \alpha \leq m<\alpha+1 \quad \text { if } p=1 .
\end{aligned}
$$

If $f$ is a measurable function on $\partial$ satisfying (1.1), then there exists a covering $\left\{r_{j}, R_{j}\right\}$ of $E(\epsilon ; \mu,(n-2) p-\beta)(\subset H)$ satisfying

$$
\sum_{j=0}^{\infty}\left(\frac{r_{j}}{R_{j}}\right)^{(n-2) p-\beta}<\infty
$$

such that

$$
\lim _{|x| \rightarrow \infty, x \in H-E(\epsilon ; \mu,(n-2) p-\beta)} N_{m}[f](x)=o\left(|x|^{1+\frac{\alpha-1}{p}} \sec ^{\frac{\beta}{p}} \theta\right) .
$$

Corollary 1 Let $1<p<\infty, n+\alpha-2>-(n-1)(p-1)$ and

$$
1-\frac{1-\alpha}{p}<m<2-\frac{1-\alpha}{p} .
$$

Iff is a measurable function on $\partial H$ satisfying (1.1), then

$$
\lim _{|x| \rightarrow \infty, x \in H} N_{m}[f](x)=o\left(|x|^{1+\frac{\alpha-1}{p}} \sec ^{n-2} \theta\right) .
$$

As an application of Theorem 1, we now show the solution of the Neumann problem with continuous data on $H$.

Theorem 2 Let $p, \beta, \alpha$ and $m$ be defined as in Theorem 1 . Iff is a continuous function on $\partial H$ satisfying (1.1), then the function $N_{m}[f]$ is a solution of the Neumann problem on $H$ with $f$ and (1.4) holds, where the exceptional set $E(\epsilon ; \mu,(n-2) p-\beta)(\subset H)$ has a covering $\left\{r_{j}, R_{j}\right\}$ satisfying (1.3).

Remark In the case $p=1, \alpha=m$ and $\beta=n-2$, then (1.3) is a finite sum and the set $E(\epsilon ; \mu, 0)$ is a bounded set. So (1.4) holds in $H$. That is to say, (1.2) holds. This is just the result of Theorem A.

Corollary 2 Let $1 \leq p<\infty, n+\alpha-2>-(n-1)(p-1)$ and

$$
\begin{aligned}
& 1-\frac{1-\alpha}{p}<m<2-\frac{1-\alpha}{p} \quad \text { if } p>1, \\
& \alpha \leq m<\alpha+1 \quad \text { if } p=1 .
\end{aligned}
$$

Iff is a continuous function on $\partial H$ satisfying (1.1), then the function $N_{m}[f]$ is a solution of the Neumann problem on $H$ with $f$ and (1.5) holds. 
The following result extends Theorem $\mathrm{B}$, which is our result in the case $p=1$ and $\alpha=m$.

Theorem 3 Let $1 \leq p<\infty, \alpha>1-p$, l be a positive integer and

$$
\begin{aligned}
& 1-\frac{1-\alpha}{p}<m<2-\frac{1-\alpha}{p} \quad \text { if } p>1 \\
& \alpha \leq m<\alpha+1 \quad \text { if } p=1 .
\end{aligned}
$$

If $f$ is a continuous function on $\partial H$ satisfying (1.1) and $h$ is a solution of the Neumann problem on $H$ with $f$ such that

$$
\lim _{|x| \rightarrow \infty, x \in H} h^{+}(x)=o\left(|x|^{l+\left[1+\frac{\alpha-1}{p}\right]}\right),
$$

then

$$
h(x)=N_{m}[f](x)+\Pi\left(x^{\prime}\right)+\sum_{j=1}^{\left[\frac{l+\left[1+\frac{\alpha-1}{p}\right]}{2}\right]} \frac{(-1)^{j}}{(2 j) !} x_{n}^{2 j} \Delta^{j} \Pi\left(x^{\prime}\right)
$$

for any $x=\left(x^{\prime}, x_{n}\right) \in H$ and $\Pi\left(x^{\prime}\right)$ is a polynomial of $x^{\prime} \in \mathbf{R}^{n-1}$ of degree less than $l+\left[1+\frac{\alpha-1}{p}\right]$.

\section{Lemmas}

In our discussions, the following estimates for the kernel function $K_{n, m}\left(x, y^{\prime}\right)$ are fundamental (see [10, Lemma 4.2] and [4, Lemmas 2.1 and 2.4]).

\section{Lemma 1}

(1) If $1 \leq\left|y^{\prime}\right| \leq \frac{|x|}{2}$, then $\left|K_{n, m}\left(x, y^{\prime}\right)\right| \lesssim|x|^{m-1}\left|y^{\prime}\right|^{-n-m+3}$.

(2) If $\frac{|x|}{2}<\left|y^{\prime}\right| \leq \frac{3}{2}|x|$, then $\left|K_{n, m}\left(x, y^{\prime}\right)\right| \lesssim\left|x-y^{\prime}\right|^{2-n}$.

(3) If $\frac{3}{2}|x|<\left|y^{\prime}\right| \leq 2|x|$, then $\left|K_{n, m}\left(x, y^{\prime}\right)\right| \lesssim x_{n}^{2-n}$.

(4) If $\left|y^{\prime}\right| \geq 2|x|$ and $\left|y^{\prime}\right| \geq 1$, then $\left|K_{n, m}\left(x, y^{\prime}\right)\right| \lesssim|x|^{m}\left|y^{\prime}\right|^{2-n-m}$.

The following lemma is due to Qiao (see [4]).

Lemma 2 If $\epsilon>0, \eta \geq 0$ and $\lambda$ is a positive measure in $\mathbf{R}^{n}$ satisfying $\lambda\left(\mathbf{R}^{n}\right)<\infty$, then $E(\epsilon ; \lambda, \eta)$ has a covering $\left\{r_{j}, R_{j}\right\}(j=1,2, \ldots)$ such that

$$
\sum_{j=1}^{\infty}\left(\frac{r_{j}}{R_{j}}\right)^{\eta}<\infty
$$

Lemma 3 ([9, Lemma 4]) Let $p, \beta, \alpha$ and $m$ be defined as in Theorem 1. Iff is a locally integral and upper semi-continuous function on $\partial H$ satisfying (1.1), then

$$
\limsup _{x \in H, x \rightarrow y^{\prime}} \frac{\partial}{\partial x_{n}} N_{m}[f](x) \leq f\left(y^{\prime}\right)
$$

for any fixed $y^{\prime} \in \partial H$. 
Lemma 4 ([2, Lemma 1]) If $h(x)$ is a harmonic polynomial of $x=\left(x^{\prime}, x_{n}\right) \in H$ of degree $m$ and $\partial h / \partial x_{n}$ vanishes on $\partial H$, then there exists a polynomial $\Pi\left(x^{\prime}\right)$ of degree $m$ such that

$$
h(x)= \begin{cases}\Pi\left(x^{\prime}\right)+\sum_{j=1}^{\left[\frac{m}{2}\right]} \frac{(-1)^{j}}{(2 j) !} x_{n}^{2 j} \Delta^{j} \Pi\left(x^{\prime}\right), & m \geq 2, \\ \Pi\left(x^{\prime}\right), & m=0,1 .\end{cases}
$$

\section{Proof of Theorem 1}

For any $\epsilon>0$, there exists $R_{\epsilon}>1$ such that

$$
\int_{\partial H\left(R_{\epsilon}, \infty\right)} \frac{\left|f\left(y^{\prime}\right)\right|^{p}}{\left(1+\left|y^{\prime}\right|\right)^{n+\alpha-2}} d y^{\prime}<\epsilon
$$

Take any point $x \in H\left(R_{\epsilon}, \infty\right)-E(\epsilon ; \mu,(n-2) p-\beta)$ such that $|x|>2 R_{\epsilon}$, and write

$$
\begin{aligned}
N_{m}[f](x) & =\left(\int_{G_{1}}+\int_{G_{2}}+\int_{G_{3}}+\int_{G_{4}}+\int_{G_{5}}\right) K_{n, m}\left(x, y^{\prime}\right) f\left(y^{\prime}\right) d y^{\prime} \\
& =U_{1}(x)+U_{2}(x)+U_{3}(x)+U_{4}(x)+U_{5}(x),
\end{aligned}
$$

where

$$
\begin{aligned}
& G_{1}=\left\{y^{\prime} \in \partial H:\left|y^{\prime}\right| \leq 1\right\}, \quad G_{2}=\left\{y^{\prime} \in \partial H: 1<\left|y^{\prime}\right| \leq \frac{|x|}{2}\right\}, \\
& G_{3}=\left\{y^{\prime} \in \partial H: \frac{|x|}{2}<\left|y^{\prime}\right| \leq \frac{3}{2}|x|\right\}, \quad G_{4}=\left\{y^{\prime} \in \partial H: \frac{3}{2}|x|<\left|y^{\prime}\right| \leq 2|x|\right\} \\
& G_{5}=\left\{y^{\prime} \in \partial H:\left|y^{\prime}\right| \geq 2|x|\right\} .
\end{aligned}
$$

First note that

$$
\begin{aligned}
\left|U_{1}(x)\right| & \lesssim \int_{G_{1}} \frac{\left|f\left(y^{\prime}\right)\right|}{\left|x-y^{\prime}\right|^{n-2}} d y^{\prime} \\
& \lesssim|x|^{2-n} \int_{G_{1}}\left|f\left(y^{\prime}\right)\right| d y^{\prime}
\end{aligned}
$$

so that

$$
\lim _{|x| \rightarrow \infty, x \in H}|x|^{-1+\frac{1-\alpha}{p}} U_{1}(x)=0 .
$$

If $m<2-\frac{1-\alpha}{p}$ and $\frac{1}{p}+\frac{1}{q}=1$, then $\left(3-n-m+\frac{n+\alpha-2}{p}\right) q+n-1>0$. By Lemma $1(1)$, (3.1) and the Hölder inequality, we have

$$
\begin{aligned}
\left|U_{2}(x)\right| & \lesssim|x|^{m-1} \int_{G_{2}}\left|y^{\prime}\right|^{-n-m+3}\left|f\left(y^{\prime}\right)\right| d y^{\prime} \\
& \lesssim|x|^{m-1}\left(\int_{G_{2}} \frac{\left|f\left(y^{\prime}\right)\right|^{p}}{\left|y^{\prime}\right|^{n+\alpha-2}} d y^{\prime}\right)^{\frac{1}{p}}\left(\int_{G_{2}}\left|y^{\prime}\right|^{\left(-n-m+3+\frac{n+\alpha-2}{p}\right) q} d y^{\prime}\right)^{\frac{1}{q}} \\
& \lesssim|x|^{1-\frac{1-\alpha}{p}}\left(\int_{G_{2}} \frac{\left|f\left(y^{\prime}\right)\right|^{p}}{\left|y^{\prime}\right|^{n+\alpha-2}} d y^{\prime}\right)^{\frac{1}{p}} .
\end{aligned}
$$


Put

$$
U_{2}(x)=U_{21}(x)+U_{22}(x)
$$

where

$$
\begin{aligned}
& U_{21}(x)=\int_{G_{2} \cap B_{n-1}\left(R_{\epsilon}\right)} K_{n, m}\left(x, y^{\prime}\right) f\left(y^{\prime}\right) d y^{\prime}, \\
& U_{22}(x)=\int_{G_{2} \backslash B_{n-1}\left(R_{\epsilon}\right)} K_{n, m}\left(x, y^{\prime}\right) f\left(y^{\prime}\right) d y^{\prime} .
\end{aligned}
$$

If $|x| \geq 2 R_{\epsilon}$, then

$$
\left|U_{21}(x)\right| \lesssim R_{\epsilon}^{2-m-\frac{1-\alpha}{p}}|x|^{m-1}
$$

Moreover, by (3.1) and (3.3), we get

$$
\left|U_{22}(x)\right| \lesssim \epsilon|x|^{1-\frac{1-\alpha}{p}}
$$

That is,

$$
\left|U_{2}(x)\right| \lesssim \epsilon|x|^{1-\frac{1-\alpha}{p}}
$$

By Lemma 1(3), (3.1) and the Hölder inequality, we have

$$
\left|U_{4}(x)\right| \lesssim \epsilon x_{n}^{2-n}|x|^{n-1-\frac{1-\alpha}{p}}
$$

If $m>1-\frac{1-\alpha}{p}$, then $\left(2-n-m+\frac{n+\alpha-2}{p}\right) q+n-1<0$. We obtain, by Lemma 1(4), (3.1) and the Hölder inequality,

$$
\begin{aligned}
\left|U_{5}(x)\right| & \lesssim|x|^{m} \int_{G_{5}}\left|y^{\prime}\right|^{-n-m+2}\left|f\left(y^{\prime}\right)\right| d y^{\prime} \\
& \lesssim|x|^{m}\left(\int_{G_{5}} \frac{\left|f\left(y^{\prime}\right)\right|^{p}}{\left|y^{\prime}\right|^{n+\alpha-2}} d y^{\prime}\right)^{\frac{1}{p}}\left(\int_{G_{5}}\left|y^{\prime}\right|^{\left(-n-m+2+\frac{n+\alpha-2}{p}\right) q} d y^{\prime}\right)^{\frac{1}{q}} \\
& \lesssim \epsilon|x|^{1-\frac{1-\alpha}{p}} .
\end{aligned}
$$

Finally, we shall estimate $U_{3}(x)$. Take a sufficiently small positive number $b$ such that $\partial H\left[\frac{|x|}{2}, \frac{3}{2}|x|\right] \subset B\left(x, \frac{|x|}{2}\right)$ for any $x \in \Pi(b)$, where

$$
\Pi(b)=\left\{x \in H ; \inf _{y^{\prime} \in \partial H}\left|\frac{x}{|x|}-\frac{y^{\prime}}{\left|y^{\prime}\right|}\right|<b\right\}
$$

and divide $H$ into two sets $\Pi(b)$ and $H-\Pi(b)$. 
If $x \in H-\Pi(b)$, then there exists a positive number $b^{\prime}$ such that $\left|x-y^{\prime}\right| \geq b^{\prime}|x|$ for any $y^{\prime} \in \partial H$, and hence

$$
\begin{aligned}
\left|U_{3}(x)\right| & \lesssim \int_{G_{3}}\left|y^{\prime}\right|^{2-n}\left|f\left(y^{\prime}\right)\right| d y^{\prime} \\
& \lesssim|x|^{m} \int_{G_{3}}\left|y^{\prime}\right|^{2-n-m}\left|f\left(y^{\prime}\right)\right| d y^{\prime} \\
& \lesssim \epsilon|x|^{1-\frac{1-\alpha}{p}}
\end{aligned}
$$

which is similar to the estimate of $U_{5}(x)$.

We shall consider the case $x \in \Pi(b)$. Now put

$$
H_{i}(x)=\left\{y^{\prime} \in \partial H\left[\frac{|x|}{2}, \frac{3}{2}|x|\right] ; 2^{i-1} \delta(x) \leq\left|x-y^{\prime}\right|<2^{i} \delta(x)\right\}
$$

where $\delta(x)=\inf _{y^{\prime} \in H}\left|x-y^{\prime}\right|$.

Since $\partial H \cap\left\{y^{\prime} \in \mathbf{R}^{n-1}:\left|x-y^{\prime}\right|<\delta(x)\right\}=\varnothing$, we have

$$
U_{3}(x)=\sum_{i=1}^{i(x)} \int_{H_{i}(x)} \frac{\left|g\left(y^{\prime}\right)\right|}{\left|x-y^{\prime}\right|^{n-2}} d y^{\prime},
$$

where $i(x)$ is a positive integer satisfying $2^{i(x)-1} \delta(x) \leq \frac{|x|}{2}<2^{i(x)} \delta(x)$.

Similar to the estimate of $U_{5}(x)$, we obtain

$$
\begin{aligned}
& \int_{H_{i}(x)} \frac{\left|g\left(y^{\prime}\right)\right|}{\left|x-y^{\prime}\right|^{n-2}} d y^{\prime} \\
& \quad \lesssim \int_{H_{i}(x)} \frac{\left|g\left(y^{\prime}\right)\right|}{\left\{2^{i-1} \delta(x)\right\}^{n-2}} d y^{\prime} \\
& \quad \lesssim \delta(x)^{\frac{\beta-(n-2) p}{p}} \int_{H_{i}(x)} \delta(x)^{\frac{(n-2) p-\beta}{p}-n+2}\left|g\left(y^{\prime}\right)\right| d y^{\prime} \\
& \quad \lesssim \cos ^{-\frac{\beta}{p}} \theta \delta(x)^{\frac{\beta-(n-2) p}{p}} \int_{H_{i}(x)}|x|^{-\frac{\beta}{p}}\left|g\left(y^{\prime}\right)\right| d y^{\prime} \\
& \quad \lesssim|x|^{n-2-\frac{\beta}{p}} \cos ^{-\frac{\beta}{p}} \theta \delta(x)^{\frac{\beta-(n-2) p}{p}} \int_{H_{i}(x)}\left|y^{\prime}\right|^{2-n}\left|g\left(y^{\prime}\right)\right| d y^{\prime} \\
& \quad \lesssim|x|^{n-1+\frac{\alpha-\beta-1}{p}} \cos ^{-\frac{\beta}{p}} \theta\left(\frac{\mu\left(H_{i}(x)\right)}{2^{i} \delta(x)^{(n-2) p-\beta}}\right)^{\frac{1}{p}}
\end{aligned}
$$

for $i=0,1,2, \ldots, i(x)$.

Since $x \notin E(\epsilon ; \mu,(n-2) p-\beta)$, we have

$$
\frac{\mu\left(H_{i}(x)\right)}{\left\{2^{i} \delta(x)\right\}^{(n-2) p-\beta}} \lesssim \frac{\mu\left(B_{n-1}\left(x, 2^{i} \delta(x)\right)\right)}{\left\{2^{i} \delta(x)\right\}^{(n-2) p-\beta}} \lesssim M(x ; \mu,(n-2) p-\beta) \lesssim \epsilon|x|^{\beta-(n-2) p}
$$

for $i=0,1,2, \ldots, i(x)-1$ and

$$
\frac{\mu\left(H_{i(x)}(x)\right)}{\left\{2^{i} \delta(x)\right\}^{(n-2) p-\beta}} \lesssim \frac{\mu\left(B_{n-1}\left(x, \frac{|x|}{2}\right)\right)}{\left(\frac{|x|}{2}\right)^{(n-2) p-\beta}} \lesssim \epsilon|x|^{\beta-(n-2) p}
$$


So

$$
\left|U_{3}(x)\right| \lesssim \epsilon|x|^{1+\frac{\alpha-1}{p}} \sec ^{\frac{\beta}{p}} \theta .
$$

Combining (3.2), (3.4)-(3.7), we obtain that if $R_{\epsilon}$ is sufficiently large and $\epsilon$ is a sufficiently small number, then $N_{m}[f](x)=o\left(|x|^{1+\frac{\alpha-1}{p}} \sec ^{\frac{\beta}{p}} \theta\right)$ as $|x| \rightarrow \infty$, where $x \in H\left(R_{\epsilon},+\infty\right)-$ $E(\epsilon ; \mu,(n-2) p-\beta)$. Finally, there exists an additional finite ball $B_{0}$ covering $H\left(0, R_{\epsilon}\right]$, which together with Lemma 2, gives the conclusion of Theorem 1.

\section{Proof of Theorem 2}

For any fixed $x \in H$, take a number $R$ satisfying $R>\max \{1,2|x|\}$. If $m>\frac{1-\alpha}{p}$, then $(2-n-$ $\left.m+\frac{n+\alpha-2}{p}\right) q+n-1<0$. By (1.1), Lemma 1(4) and the Hölder inequality, we have

$$
\begin{aligned}
& \int_{\partial H(R, \infty)}\left|K_{n, m}\left(x, y^{\prime}\right)\right|\left|f\left(y^{\prime}\right)\right| d y^{\prime} \\
& \quad \lesssim|x|^{m} \int_{\partial H(R, \infty)}\left|y^{\prime}\right|^{2-n-m}\left|f\left(y^{\prime}\right)\right| d y^{\prime} \\
& \quad \lesssim|x|^{m}\left(\int_{\partial H(R, \infty)} \frac{\left|f\left(y^{\prime}\right)\right|^{p}}{\left|y^{\prime}\right|^{n+\alpha-2}} d y^{\prime}\right)^{\frac{1}{p}}\left(\int_{\partial H(R, \infty)}\left|y^{\prime}\right|^{\left(-n-m+2+\frac{n+\alpha-2}{p}\right) q} d y^{\prime}\right)^{\frac{1}{q}} \\
& <\infty .
\end{aligned}
$$

Hence $N_{m}[f](x)$ is absolutely convergent and finite for any $x \in H$. Thus $N_{m}[f](x)$ is harmonic on $H$.

To prove

$$
\lim _{x \rightarrow y^{\prime}, x \in H} \frac{\partial}{\partial x_{n}} N_{m}[f](x)=f\left(y^{\prime}\right)
$$

for any point $y^{\prime} \in \partial H$, we only need to apply Lemma 3 to $f(y)$ and $-f(y)$.

We complete the proof of Theorem 2.

\section{Proof of Theorem 3}

Consider the function $h^{\prime}(x)=h(x)-N_{m}[f](x)$. Then it follows from Theorems 2 and 3 that $h^{\prime}(x)$ is a solution of the Neumann problem on $H$ with $f$ and it is an even function of $x_{n}$ (see [2, p.92]).

Since

$$
0 \leq\left\{h-N_{m}[f]\right\}^{+}(x) \leq h^{+}(x)+\left\{N_{m}[f]\right\}^{-}(x)
$$

for any $x \in H$, and

$$
\lim _{|x| \rightarrow \infty, x \in H} N_{m}[f](x)=o\left(|x|^{1+\frac{\alpha-1}{p}}\right)
$$

from Theorem 2.

Moreover, (1.6) gives that

$$
\lim _{|x| \rightarrow \infty, x \in H}\left(h-N_{m}[f]\right)(x)=o\left(|x|^{l+\left[1+\frac{\alpha-1}{p}\right]}\right) .
$$


This implies that $h^{\prime}(x)$ is a polynomial of degree less than $l+\left[1+\frac{\alpha-1}{p}\right]$ (see [11, Appendix]), which gives the conclusion of Theorem 3 from Lemma 4.

\section{Competing interests}

The authors declare that there is no conflict of interests regarding the publication of this article.

\section{Authors' contributions}

All authors contributed equally to the manuscript and read and approved the final manuscript.

\section{Author details}

${ }^{1}$ Department of Mathematics and Information Science, Henan University of Economics and Law, Zhengzhou, 450002, P.R. China. ${ }^{2}$ College of Applied Mathematics, Chengdu University of Information Technology, Chengdu, 610225, P.R. China.

\section{Acknowledgements}

The authors are thankful to the referees for their helpful suggestions and necessary corrections in the completion of this paper

Received: 13 August 2013 Accepted: 23 October 2013 Published: 04 Dec 2013

\section{References}

1. Szegö, G: Orthogonal Polynomials. American Mathematical Society: Colloquium Publications, vol. 23. Am. Math. Soc., Providence (1975)

2. Armitage, $\mathrm{DH}$ : The Neumann problem for a function in $\mathrm{R}^{n} \times(0, \infty)$. Arch. Ration. Mech. Anal. 63, $89-105$ (1976)

3. Huang, JJ, Qiao, L: The Dirichlet problem on the upper half-space. Abstr. Appl. Anal. 2012, Article ID 203096 (2012)

4. Qiao, L: Modified Poisson integral and Green potential on a half-space. Abstr. Appl. Anal. 2012, Article ID 765965 (2012)

5. Siegel, D, Talvila, E: Sharp growth estimates for modified Poisson integrals in a half space. Potential Anal. 15(4), 333-360 (2001)

6. Qiao, L, Deng, GT: Growth estimates for modified Green potentials in the upper-half space. Bull. Sci. Math. 135, 279-290 (2011)

7. Armitage, DH: On harmonic polynomials. Proc. Lond. Math. Soc. 38, 53-71 (1979)

8. Su, BY: Dirichlet problem for the Schrödinger operator in a half space. Abstr. Appl. Anal. 2012, Article ID 578197 (2012)

9. Su, BY: Growth properties of harmonic functions in the upper half space. Acta Math. Sin. 55(6), 1095-1100 (2012) (in Chinese)

10. Hayman, WK, Kennedy, PB: Subharmonic Functions, vol. 1. Academic Press, London (1976)

11. Brelot, M: Éléments de la théorie classique du potential, pp. 53-71. Centre de Documentation Universitaire, Paris (1965)

10.1186/1029-242X-2013-572

Cite this article as: Ren and Yang: Growth estimates for modified Neumann integrals in a half-space. Journal of Inequalities and Applications 2013, 2013:572

\section{Submit your manuscript to a SpringerOpen ${ }^{\circ}$ journal and benefit from:}

- Convenient online submission

- Rigorous peer review

- Immediate publication on acceptance

- Open access: articles freely available online

- High visibility within the field

- Retaining the copyright to your article 